\title{
I misremember it well: Why older adults are unreliable eyewitnesses
}

\author{
CHAD S. DODSON and LACY E. KRUEGER \\ University of Virginia, Charlottesville, Virginia
}

\begin{abstract}
We used the eyewitness suggestibility paradigm to investigate the hypothesis that cognitive aging is associated with an increase in misrecollections - confidently held but false memories of past events. When younger and older adults were matched on their overall memory for experienced events, both groups showed comparable rates of suggestibility errors in which they claimed to have seen events in a video that had only been suggested in a subsequent questionnaire. However, older adults werealarmingly_most likely to commit suggestibility errors when they were most confident about the correctness of their response. By contrast, their younger, accuracy-matched counterparts were most likely to commit these errors when they were uncertain about the accuracy of their response. The elderly adults' propensity to make high-confidence errors fits our misrecollection account.
\end{abstract}

In the film Gigi, an elderly man recounts a long-ago love affair in the song, "I Remember It Well." What is remarkable about his description of the event is not only the huge number of details he apparently misremembers, but also the confidence with which he continues to produce more misrecollections.

Research in cognitive aging has long recognized that older adults are more susceptible to false memories - both "remembering" events that never occurred and misremembering events that did occur- than are younger adults (for general reviews, see Dodson, Koutstaal, \& Schacter, 2000; Johnson, Hashtroudi, \& Lindsay, 1993; Roediger, 1996; Schacter, Koutstaal, \& Norman, 1997). Currently, the prevailing account for this susceptibility is that older adults have greater difficulty recollecting or using source information - specific item information about an event, such as when and where it occurred (e.g., Balota, Dolan, \& Duchek, 2000; Dodson \& Schacter, 2002; Johnson et al., 1993; Memon, Bartlett, Rose, \& Gray, 2003; Schacter, Norman, \& Koutstaal, 1998). Failing to remember or use source information that identifies how a particular person or event was encountered means that the elderly must more often guess or rely on overall familiarity as a basis for a response, which can lead to memory errors.

We investigated an alternative, but not necessarily competing, hypothesis about why aging is associated with increased memory distortions. According to the misrecollection account, the elderly are prone to making high-

We thank Amina Memon, Karen Mitchell, Roddy Roediger, Bill Rohwer, and Bobbie Spellman for their helpful comments. We are also grateful for the support from the Institute of Aging at the University of Virginia. Correspondence concerning this article should be addressed to C. S. Dodson, Department of Psychology, University of Virginia, P.O. Box 400400, 102 Gilmer Hall, Charlottesville, VA 22904-4400 (e-mail: cdodson@virginia.edu). confidence errors when answering questions about specific details of recently learned events. Our misrecollection account builds on the ideas of others that explain age-related memory impairments in terms of changes in the capacity to bind together and associate different items or features of events (e.g., Chalfonte \& Johnson, 1996; Henkel, Johnson, \& De Leonardis, 1998; Koutstaal, Schacter, \& Brenner, 2001; Kroll, Knight, Metcalfe, Wolf, \& Tulving, 1996; Schacter et al., 1998). For instance, Henkel et al. suggested that older adults are susceptible to miscombining features of one event with features of other events, which contributes to misremembering how events occurred (see also Koutstaal et al., 2001; Kroll et al., 1996). We suggest that the kind of feature miscombinations that occur in the elderly produce convincing misrecollections, which, in turn, lead to high-confidence errors (for more details, see Dodson, Bawa, \& Krueger, in press).

We used an eyewitness suggestibility paradigm to evaluate the misrecollection hypothesis. Participants watched a video clip of a crime and then answered questions about the witnessed event. Some questions referred to details that were never actually witnessed in the video, such as that the police said, "we'll shoot!" or that the burglar had a gun. The participants then completed a source memory test that contained items that referred to events that had been seen in the video, read in the questionnaire, both seen and read, or not encountered in the experiment (i.e., new items). The participants were clearly informed that the questionnaire referred to some events that had not occurred in the video and that they should indicate for each test item whether it had been encountered in the video only, the questionnaire only, both, or neither. After making a response, they then assessed its likely accuracy.

Many studies of eyewitness memory have shown that older adults are prone to claiming to have witnessed events that were only suggested to them and that they also tend to express higher confidence in these false memories 
than do younger adults (e.g., Cohen \& Faulkner, 1989; Loftus, Levidow, \& Duensing, 1992; Mitchell, Johnson, \& Mather, 2003; Mueller-Johnson \& Ceci, 2004; for a review, see Bartlett \& Memon, in press). However, as Jacoby and colleagues have noted, these age-related differences may simply reflect older adults' reduced memory for what was learned, just as the age-related vulnerability to retroactive and proactive interference has been greatly reduced when older and younger adults have been matched on degree of initial learning (Jacoby, Bishara, Hessels, \& Toth, 2005). No study has attempted to identify the cause of the age-related suggestibility effect by equating correct source identification performance between younger and older adults. Moreover, given the influence of eyewitness confidence on jury decision making, there is much practical importance in determining how well younger and older adults can monitor the accuracy of their memories by assigning low confidence to suggestibility errors.

A critical test of the misrecollection hypothesis involves, first, equating correct source identification performance between younger and older adults. If the misrecollection account is correct that the elderly are disproportionately susceptible to making high-confidence errors, older adults should express high confidence in the accuracy of a greater proportion of false memories than will younger adults who make the same overall amount of false memories. There are potentially enormous practical and policy implications from this prediction, because it indicates that the elderly may be prone to being incorrect when they are most confident in the accuracy of their memory.

We compared three groups of participants. We included a group of young and older adults who received the identical conditions, in order to verify that our study replicates past findings of increased false memories in older adults. However, the central test of our prediction required that we equate source identification performance between older and younger adults. To accomplish this, we included an additional group of young participants who received a 2-day delay between answering the questionnaire and receiving the final memory test, in order to match their source memory with that of the older participants.

\section{METHOD}

\section{Participants}

Seventy-two undergraduates (17-23 years of age) were assigned to either the young or the young-delay condition, and 36 healthy older adults (60-79 years) with no known cognitive deficiencies ${ }^{1}$ were assigned to the older condition. Table 1 displays demographic information for all the participants. The individuals in the older group had completed significantly more years of schooling than had the individuals in each of the other groups $\left[F(2,105)=30.17, M S_{\mathrm{e}}=\right.$ 2.92; all $p \mathrm{~s}<.05$, as determined by Fisher's PLSD post hoc tests]. For the logical memory and vocabulary tests, we assessed the representativeness of our samples by analyzing scaled scores (Wechsler, 1997a, 1997b; scaled scores reflect an individual's performance, relative to the typical score of their age range, with a scaled score of 10 corresponding to average performance). ${ }^{2}$ The three groups did not differ on either their logical memory scaled scores, $[F(2,105)=$ $1.04]$ or their vocabulary scaled scores $[F(2,105)=1.15]$. Thus, the groups were comparably representative of their age groups.

\section{Materials and Procedure}

The participants were tested individually. The stimuli, based on those used by Mitchell et al. (2003), were presented on a 17-in. monitor located approximately $51 \mathrm{~cm}$ away from the participants. The participants watched a 5-min video reenacting a burglary and police chase. Immediately afterward, they answered 24 yes-no questions about the video, of which 8 items contained misleading suggestions. For example, in the video, the police officers did not threaten to shoot at the burglar, but some participants received misinformation in the questionnaire suggesting that they had. Specifically, one misleading questionnaire item asked, "When the police said, 'Pull over or we'll shoot! You're under arrest,' did the driver pull over?" The participants who were not misled for this item were asked, "When the police said, 'Pull over! You're under arrest,' did the driver pull over?" Misleading questions were intermixed with nonmisleading questions. The eight misleading suggestions were drawn from a pool of 12 critical items. The remaining 4 items not presented in the questionnaire were used as control (i.e., new) items in the source memory test. Three versions of the questionnaire were created to counterbalance the 4 new items and 8 misled items, so that across participants, all 12 critical items served as misled items and new items. Following the questionnaire, the young and older participants completed an unrelated spatial relations task for $10 \mathrm{~min}$. However, to match overall memory between the young-delay and the older groups, the participants in the youngdelay group departed after the questionnaire phase and returned 2 days later to complete the source memory test.

This final source memory test was unexpected and consisted of 32 visually presented statements: 8 referred to events that were shown in the video only, 8 referred to events that were presented only in the questionnaire (the misled items), 8 referred to events that were shown in both the video and the questionnaire, and 8 referred to new events that were never shown ( 4 control items and 4 filler items). The participants were clearly informed that the questionnaire referred to some events that actually had not occurred in the video. They were told that the statements on the source memory test referred to events that were experienced in one of four possible ways, corresponding to four response options: "video only," "questionnaire only," "both video and questionnaire," or "neither video nor questionnaire."

Table 1

Demographic Information for Younger and Older Adults

\begin{tabular}{|c|c|c|c|c|c|c|c|c|c|c|c|c|}
\hline \multirow[b]{3}{*}{ Group } & \multirow{3}{*}{$\begin{array}{c}\text { Age } \\
\text { (years) }\end{array}$} & \multirow{3}{*}{$\begin{array}{c}\% \\
\text { Female } \\
\end{array}$} & \multirow{2}{*}{\multicolumn{2}{|c|}{$\begin{array}{c}\text { Years of } \\
\text { Education }\end{array}$}} & \multicolumn{4}{|c|}{$\begin{array}{c}\text { Logical } \\
\text { Memory }\end{array}$} & \multicolumn{4}{|c|}{$\begin{array}{c}\text { WAIS-III } \\
\text { Vocabulary }\end{array}$} \\
\hline & & & & & \multicolumn{2}{|c|}{ Raw } & \multicolumn{2}{|c|}{ Scaled } & \multicolumn{2}{|c|}{ Raw } & \multicolumn{2}{|c|}{ Scaled } \\
\hline & & & $M$ & $S E M$ & $M$ & SEM & $M$ & $S E M$ & $M$ & SEM & $M$ & $S E M$ \\
\hline Young & 18.7 & 61 & 13.2 & 0.18 & 53.3 & 1.5 & 13.1 & 0.47 & 51.8 & 1.1 & 14.3 & 0.40 \\
\hline Young-delay & 19.7 & 67 & 13.9 & 0.25 & 55.7 & 1.5 & 13.8 & 0.44 & 50.7 & 1.2 & 13.9 & 0.42 \\
\hline Older & 68.1 & 58 & 16.2 & 0.38 & 44.5 & 1.5 & 12.9 & 0.46 & 53.9 & 1.2 & 13.4 & 0.39 \\
\hline
\end{tabular}

Note-Logical Memory is a subset of the Wechsler Memory Scale, and the WAIS-III Vocabulary is a subset of the Wechsler Adult Intelligence Scale-III. 
They were instructed to select a response on the basis of their own memory for the event and then to rate the likely accuracy of their response, using the following scale: 50 (guessing), 60, 70, 80, 90, or 100 (certain). Next, the participants were given the option of either submitting or withholding their response for scoring. However, these data are not presented, because of space constraints and the lack of an effect on either the source accuracy or the suggestibility scores. Finally, the participants completed the vocabulary and logical memory subtests of the WAIS-III and the Wechsler Memory scale, respectively, which are standard tests of cognitive functioning.

\section{RESULTS}

\section{Matching Recognition and Source Identification Performance}

Overall, we were successful in matching recognition and source identification performance between the older and the young-delay groups. Table 2 displays the rate at which older, young-delay, and young adults responded "video," "questionnaire," "both," and "new" to test items from these four different sources. For item recognition, we examined hit rates and false alarm rates by computing the sum of responses of "video," "questionnaire," and "both" to the studied and new items, respectively. Because only the critical items were counterbalanced between appearing in the questionnaire and appearing as new items on the test, we analyzed corrected recognition rates for these items by subtracting the false alarm rate to the new items from the hit rate to the questionnaire items. There were group differences in corrected recognition rates for the questionnaire items $\left[F(2,105)=10.73, M S_{\mathrm{e}}=0.08, p<.01\right]$. Young (.67) individuals showed higher corrected recognition rates than did either the older (.39) or the young-delay (.40) individuals ( $p \mathrm{~s}<.01$, as determined by Fisher's PLSD post hoc tests). The older and the young-delay adults showed nearly identical corrected recognition rates. However, as can be seen in Table 2, there were substantial group differences in false alarm rates to the new items $[F(2,105)=$

Table 2

Rate of Responding "Video," "Questionnaire," "Both," and "New" to Test Items From These Four Different Sources by the Younger, Young-Delay, and Older Groups

\begin{tabular}{|c|c|c|c|c|}
\hline \multirow[b]{2}{*}{ Item Type } & \multicolumn{4}{|c|}{ Response } \\
\hline & "Video" & "Questionnaire" & "Both" & "New" \\
\hline \multicolumn{5}{|c|}{ Younger Adults } \\
\hline Video & .85 & .01 & .04 & .10 \\
\hline Questionnaire & .09 & .56 & .16 & .19 \\
\hline Both & .27 & .15 & .52 & .06 \\
\hline New & .10 & .03 & .01 & .86 \\
\hline \multicolumn{5}{|c|}{ Young-Delay Adults } \\
\hline Video & .68 & .02 & .12 & .18 \\
\hline Questionnaire & .20 & .32 & .13 & .35 \\
\hline Both & .41 & .06 & .42 & .11 \\
\hline New & .22 & .01 & .02 & .75 \\
\hline \multicolumn{5}{|c|}{ Older Adults } \\
\hline Video & .72 & .03 & .14 & .11 \\
\hline Questionnaire & .16 & .28 & .08 & .48 \\
\hline Both & .50 & .06 & .36 & .09 \\
\hline New & .10 & .02 & .01 & .87 \\
\hline
\end{tabular}

Note-Correct responses are in bold.
3.24, $\left.M S_{\mathrm{e}}=0.05, p<.05\right]$, with the young-delay adults (.25) showing significantly larger false alarm rates than did either the older $(.13)$ or the younger $(.14)$ adults $(p \mathrm{~s}<$ $.05)$; the latter two groups did not differ from each other. As for recognition of the remaining item types, there were significant group differences in hit rates to the video items $\left[F(2,105)=6.30, M S_{\mathrm{e}}=0.01, p<.01\right]$, with the older (.89) and the younger (.90) groups showing comparable hit rates and both showing significantly higher hit rates than did the young-delay $(.82)$ group $(p \mathrm{~s}<.01)$. There were no significant group differences in the hit rates for the both items $[F(2,105)=2.22]$.

We analyzed source memory performance by computing conditional source identification scores for each item type. These scores measure an individual's ability to identify the correct source of an item when he or she recognizes it as having been encountered previously. ${ }^{3}$ The overall pattern across the groups paralleled that of the recognition data: no difference between the older and the young-delay groups and superior performance by the young group. All ANOVAs of the source scores for the video items, both items, and questionnaire items showed significant group effects (all $F_{\mathrm{S}}>7.47$, all $p \mathrm{~s}<.01$ ). For each item type, the young showed significantly higher source scores than did the other two groups (all $p \mathrm{~s}<.05$, as determined by Fisher's PLSD post hoc tests). Critically, the older and the young-delay groups showed comparable source scores for all the items and did not differ significantly.

Moreover, because our central prediction depends on matching performance between the older and the youngdelay groups, we fit two high-threshold multinomial models to each of these groups' data-specifically, the model designed by Dodson and Shimamura (2000, Experiment 2) for three study sources. There were no significant differences between these groups on any of the parameter values that correspond to source memory for the different item types [all $G^{2}(1) \mathrm{s}<2.45, p s>.10$ ]. For the parameter values corresponding to item recognition, older and young-delay adults showed comparable recognition memory for the questionnaire items, and older adults showed superior recognition memory for the video and both items [all $G^{2}(1) \mathrm{s}>3.72, p \mathrm{~s}<.055$ ]. Lastly, there were no differences between the young-delay and the older adults on the parameter values corresponding to the response bias to commit suggestibility errors - that is, the overall tendency to respond "video" and "both" [all $\left.G^{2}(1) \mathrm{s}<0.5\right]$. Due to space constraints and their redundancy with the recognition and conditional source scores, we will not describe these model-based results in more detail.

Overall, then, the matching procedure was successful. In particular, for the critical questionnaire items, the older and the young-delay groups showed nearly identical corrected recognition and source identification scores.

\section{Suggestibility Errors: Claiming to Have Witnessed Suggested Details}

Two types of suggestibility errors were possible for the items that were only read about in the questionnaire (i.e., suggested items). Individuals could claim to have seen a 
suggested item in the video only, or they could claim that a suggested item occurred in both the video and the questionnaire. Following prior studies, we will use the term suggestibility error to refer to the combined proportion of both kinds of errors (e.g., Mitchell et al., 2003).

The older and young-delay individuals were more suggestible than the younger individuals. An ANOVA of the proportion of questionnaire items recognized as old that were incorrectly labeled either "video only" or "both" (i.e., total suggestibility errors) revealed a significant main effect of group $\left[F(2,103)=7.04, M S_{\mathrm{e}}=0.10, p<\right.$ $.01]$. The young (.31) adults committed fewer suggestibility errors than did either the older (.55) or the young-delay (.54) adults $(p \mathrm{~s}<.01$, as determined by Fisher's PLSD post hoc tests). The older and the young-delay adults were comparably suggestible. Moreover, when the two kinds of suggestibility errors are considered separately, incorrectly responding "video" made up a significantly higher proportion of the overall amount of suggestibility errors for both the older adults (.64) and the young-delay adults (.61) than it did for the younger adults $(.40)(p \mathrm{~s}<.05)$. Thus, as others have observed, time delays in younger adults and advanced age are associated with an increased vulnerability to claiming to have seen items that were only read about (e.g., Higham, 1998; Mitchell et al., 2003).

Although the multinomial modeling analysis indicated no differences between the young-delay and the older adults in their overall response bias to commit suggestibility errors, we further analyzed possible differences in response bias by examining the tendency to respond "video" and "both" to new items that were mistakenly considered as having been encountered during the experiment. Although the young-delay adults made more false alarms overall than did the older and younger individuals, as can be seen in Table 2, the proportion of these false alarms that were assigned to the video and both sources was comparable across the three groups $[F(2,48)<1]$. Therefore, there appear to be no group differences in the bias to make suggestibility errors.

It is important to emphasize that there is a confound in the traditional method of analyzing suggestibility errors. Suggestibility effects have been assumed to occur when individuals show a rate of responding "video" or "both" to recognized questionnaire items that is greater than the raw rate of responding in this manner to new items. Responding "video" or "both" to new items is assumed to reflect the baseline tendency (or response bias) to make suggestibility errors. The problem with this analysis is that the raw rate of responding "video" or "both" to new items is influenced by two different factors: (1) an individual's bias to respond in this manner and (2) an individual's overall false alarm rate. For example, an examination of Table 2 shows that our young-delay individuals might appear to have a stronger suggestibility response bias than do the other groups, given their larger rate of responding "video" (.22) and "both" (.02) to the new items. However, these large response rates are merely a by-product of the young-delay group's larger overall false alarm rate (.25). When this overall false alarm rate is taken into account- by examining the proportion of false alarms that are called "video" and "both"- there are no substantial differences between the groups in the tendency to respond "video" and "both." In short, the standard analysis is confounded whenever there are group differences in the overall false alarm rate.

\section{When Matched With Young on Source Memory, Are the Elderly More Likely to be Incorrect When They Are Most Confident?}

Our central prediction is that older adults are more likely than their accuracy-matched younger counterparts to be wrong about having seen a suggested item when they are certain about the correctness of their response. For this evaluation, we sorted the data into three groupings based on individuals' estimates of the likely accuracy of their response: Accuracy ratings of 50\%/60\% were labeled low confidence, accuracy ratings of $70 \% / 80 \%$ were labeled medium confidence, and accuracy ratings of $90 \% / 100 \%$ were labeled high confidence. We then examined the proportions of suggestibility errors - responding either "video" or "both" to recognized questionnaire items - at each confidence level, which are displayed in Figure 1.

As is clear in the figure, the tendency to commit suggestibility errors is positively correlated with confidence in older adults but negatively correlated with confidence in young-delay adults. Because the proportions of suggestibility errors across the low-, medium-, and highconfidence groupings must sum to one, these three rates are not independent of each other. Consequently, given the assumptions of the ANOVA, we analyzed the proportions of suggestibility errors to the questionnaire items at the low- and high-confidence levels, since responses at two of these confidence levels are independent of each other.

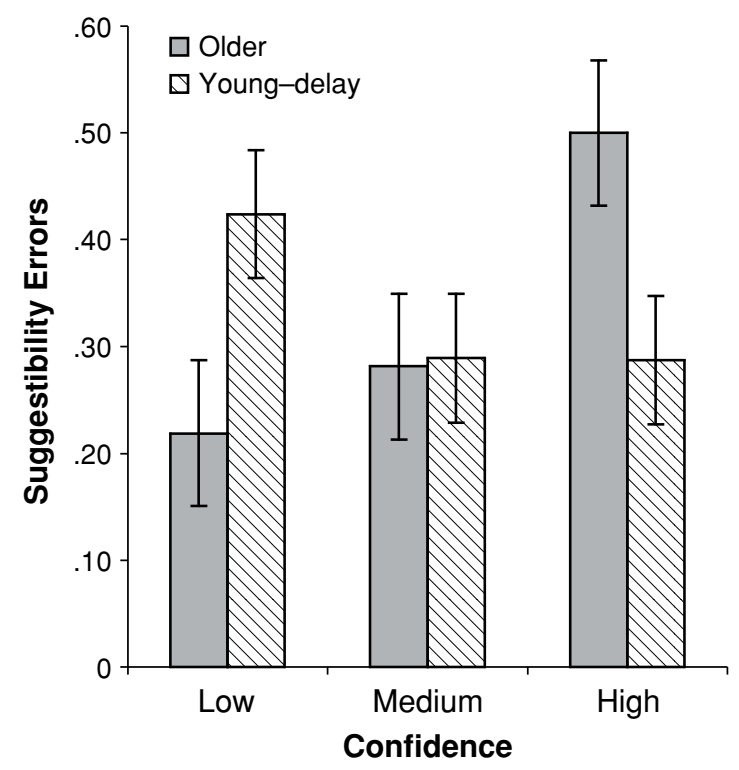

Figure 1. Proportion of suggestibility errors at each confidence level for Older and Young-Delay adults. Error bars are standard errors of the means. 
A 2 (confidence: low vs. high) $\times 2$ (age: young-delay vs. older adults) ANOVA yielded a significant interaction $\left[F(1,63)=6.58, M S_{\mathrm{e}}=0.22, p=.01\right]$ and no other significant effects $($ all $F \mathrm{~s}<1) .{ }^{4}$

As can be seen in Figure 1, the interaction is fueled by the young-delay and the older adults' opposite pattern of responding. The older adults gave high-confidence responses to nearly twice as many suggestibility errors as did the young-delay adults $[t(63)=2.31, p<.05]$. By contrast, the young-delay adults gave low-confidence responses to nearly twice as many suggestibility errors as did the older adults $[t(63)=2.29, p<.05]$. Moreover, these findings are not driven by a few highly suggestible individuals. We split each age group into halves on the basis of their overall rate of making suggestibility errors to the questionnaire items and included this variable in the foregoing ANOVA. A 2 (age: young-delay vs. older) $\times$ 2 (overall suggestibility rate: good vs. poor) $\times 2$ (confidence: low vs. high) showed only a significant age $\times$ confidence interaction $\left[F(1,61)=6.86, M S_{\mathrm{e}}=0.22, p<.05\right]$ and no other significant effects (all $F \mathrm{~s}<1.09$ ). In other words, the same pattern of assigning confidence ratings to suggestibility errors occurred when both age groups made either many or few suggestibility errors overall.

Lastly, these differences are not driven by an age-related shift to respond confidently to all incorrect responses of claiming to have either seen or both seen and read items. That is, both the young-delay and the older adults gave similar proportions of low-confidence (.43 and .48, respectively) and high-confidence (.31 and .29 , respectively) responses to new items that were mistakenly thought of as having been either seen or both seen and read earlier.

\section{DISCUSSION}

When the younger and the older adults were matched on their overall memory for experienced events, both groups showed comparable rates of suggestibility errors in which they claimed to have seen events in a video that had only been suggested in a subsequent questionnaire. Strikingly, the older adults most often made these suggestibility errors when they were certain about the correctness of their response. By contrast, their younger, accuracymatched counterparts were most likely to make these errors when they were uncertain about the correctness of their response. These findings are of both theoretical and practical importance.

Theoretically, these findings fit the predictions of our misrecollection hypothesis. Advancing age is associated with an increased susceptibility of miscombining features of different events, such as features from objects that were seen with features of objects that were only read about. However, the essential and novel component of our account is that miscombined features are sufficiently bound together that they produce phenomenal experiences that are as confidently held as vivid true memories. It is the latter component that predicts that the elderly are prone to showing false memories when they are certain about the accuracy of their memory. Moreover, our misrecollection account suggests that in other paradigms, the increased occurrence of memory errors by older adults may not be caused by an age-related increase in guessing. Instead, we suggest that these false memory errors reflect, to some extent, elderly adults' tendency to confidently misremember past events.

Practically, the present results have disconcerting implications for the trustworthiness of older adults' eyewitness testimony. Alarmingly, older adults showed the largest proportion of suggestibility errors when they were quite certain about the accuracy of their responses. This pattern of behavior is particularly worrisome, given the influence of eyewitness confidence on jury decision making. Further research is critical for determining the generality of our findings, since some studies have shown no age-related differences in the overall magnitude of the suggestibility effect, although no other study has been performed to examine how the tendency to make suggestibility errors varies with changes in confidence (e.g., Coxon \& Valentine, 1997; Gabbert, Memon, \& Allan, 2003; see Bartlett \& Memon, in press, for a review). Finally, a favorable result emerged in the performance of the younger adults in the delay condition. Despite their poor memory for what they encountered, these individuals accurately assessed the reliability of their responses and assigned low confidence to the largest proportion of their suggestibility errors.

\section{REFERENCES}

Balota, D. A., Dolan, P. O., \& Duchek, J. M. (2000). Memory changes in healthy older adults. In E. Tulving and F. I. M. Craik (Eds.), The Oxford handbook of memory (pp. 395-410). Oxford: Oxford University Press.

Bartlett, J. C., \& Memon, A. (in press). Eyewitness memory in young and older adults. In R. C. L. Lindsay, D. F. Ross, J. D. Read, \& M. P. Toglia (Eds.), The handbook of eyewitness psychology: Vol. II. Mahwah, NJ: Erlbaum.

Chalfonte, B. L., \& Johnson, M. K. (1996). Feature memory and binding in young and older adults. Memory \& Cognition, 24, 403-416.

Cohen, G., \& FaulKner, D. (1989). Age differences in source forgetting: Effects on reality monitoring and on eyewitness testimony. Psychology \& Aging, 4, 10-17.

Coxon, P., \& Valentine, T. (1997). The effects of the age of eyewitnesses on the accuracy and suggestibility of their testimony. Applied Cognitive Psychology, 11, 415-430.

Dodson, C. S., Bawa, S., \& Krueger, L. E. (in press). Aging, metamemory and high confidence errors: A misrecollection account. Psychology \& Aging.

Dodson, C. S., Koutstaal, W., \& Schacter, D. L. (2000). Escape from illusion: Reducing false memories. Trends in Cognitive Sciences, $\mathbf{4}$, 391-397.

Dodson, C. S., \& Schacter, D. L. (2002). Aging and strategic retrieval processes: Reducing false memories with a distinctiveness heuristic. Psychology \& Aging, 17, 405-415.

Dodson, C. S., \& Shimamura, A. P. (2000). Differential effects of cue dependency on item and source memory. Journal of Experimental Psychology: Learning, Memory, \& Cognition, 26, 1023-1044.

Gabbert, F., Memon, A., \& Allan, K. (2003). Memory conformity: Can eyewitnesses influence each other's memories for an event? Applied Cognitive Psychology, 17, 533-544.

Henkel, L. A., Johnson, M. K., \& De Leonardis, D. M. (1998). Aging and source monitoring: Cognitive processes and neuropsychological correlates. Journal of Experimental Psychology: General, 127, 251-268.

Higham, P. A. (1998). Believing details known to have been suggested. British Journal of Psychology, 89, 265-279.

Jacoby, L. L., Bishara, A. J., Hessels, S., \& Toth, J. P. (2005). Aging, 
subjective experience, and cognitive control: Dramatic false remembering by older adults. Journal of Experimental Psychology: General, 134, 131-148.

Johnson, M. K., Hashtroudi, S., \& Lindsay, D. S. (1993). Source monitoring. Psychological Bulletin, 114, 3-28.

Koutstaal, W., Schacter, D. L., \& Brenner, C. (2001). Dual task demands and gist-based false recognition of pictures in younger and older adults. Journal of Memory \& Language, 44, 399-426.

Kroll, N. E. A., Knight, R. T., Metcalfe, J., Wolf, E. S., \& Tulving, E. (1996). Cohesion failure as a source of memory illusions. Journal of Memory \& Language, 35, 176-196.

Loftus, E. F., Levidow, B., \& Duensing, S. (1992). Who remembers best? Individual differences in memory for events that occurred in a science museum. Applied Cognitive Psychology, 6, 93-107.

Memon, A., Bartlett, J., Rose, R., \& Gray, C. (2003). The aging eyewitness: Effects of age on face, delay and source-memory ability. Journals of Gerontology, 58B, P338-P345.

Mitchell, K. J., Johnson, M. K., \& Mather, M. (2003). Source monitoring and suggestibility to misinformation: Adult age-related differences. Applied Cognitive Psychology, 17, 107-119.

Mueller-Johnson, K., \& CeCI, S. J. (2004). Memory and suggestibility in older adults: Live event participation and repeated interview. Applied Cognitive Psychology, 18, 1109-1127.

RoEDIGER, H. L., III (1996). Memory illusions. Journal of Memory \& Language, 35, 76-100.

Schacter, D. L., Koutstaal, W., \& Norman, K. A. (1997). False memories and aging. Trends in Cognitive Sciences, 1, 229-236.

Schacter, D. L., Norman, K. A., \& Koutstaal, W. (1998). The cog- nitive neurosciences of constructive memory. Annual Review of Psychology, 49, 289-318.

WECHSLER, D. (1997a). Wechsler Adult Intelligence Scale-Third edition. San Antonio, TX: Psychological Corporation.

WeChSLER, D. (1997b). Wechsler Memory Scale-Third edition. San Antonio, TX: The Psychological Corporation.

\section{NOTES}

1. Older adults were asked whether they had had any sort of disease or accident that might have impaired their memory or cognitive functioning in any way, such as a stroke, Alzheimer's disease, Parkinson's disease, and so forth

2. Given the reliable age-related decrement in measures of fluid intelligence (e.g., logical memory test) and increment in measures of crystallized intelligence (e.g., vocabulary test), we believe that it is most effective to match samples on the basis of their scaled scores, as opposed to their raw scores. However, we provide the raw scores in Table 1.

3. Two older adults recognized none of the questionnaire items (i.e., they responded "new" to all of these items). Consequently, both the questionnaire source scores and the suggestibility scores for these 2 individuals are undefined, because computing both of these scores depends on recognizing the item as previously encountered.

4 . Four older adults and 3 young-delay adults committed no suggestibility errors and, thus, could not be included in this analysis.

(Manuscript received August 29, 2005; revision accepted for publication January 31,2006 .) 